\title{
El denominador "nacidos vivos" \\ en investigación de salud pública
}

Estuardo Albán, César Hermida, Jorge Hermida, juan Vásconez, Mario Vergara, Miguel Hinojasa,

Miguel Machuca, Nelson Oviedo

REDSISMLL', Ecuador

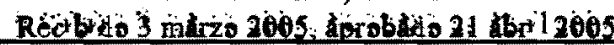

\begin{abstract}
RESUMEN: Con el objetivo de ajustar los datos nacionales y mostrar la metodología para ajustes provinciales y locales, se calculan las inscripciones tardías de nacidos vivos a partir de la información del Instituto de Estadísticas y Censos. Se obtiene un denominador más apropiado para los cálculos de mortalidad infantil y materna, y las estimaciones de coberturas del programa de vacunación y otros. Se compara este dato con el número total de inscripciones en el año, para destacar la inconveniencia de su uso, así como la inconveniencia de optar por las proyecciones del número de menores de un año como altemativa para la estimación de nacidos vivos.
\end{abstract}

PALABRAS CLAVES: Nacidos vivos, mortalidad infantil, mortalidad materna, coberturas de vacunación.

\section{INTRODUCCIÓN:}

En el año 2000 los 189 Estados Miembros de las Naciones Unidas se reunieron en la Cumbre del Milenio y aprobaron ocho objetivos y 18 metas para combatir la pobreza, el hambre, las enfermedades, la discriminación contra la mujer, la degradación de las tierras y el analfabetismo. El Objetivo 4 propone "reducir la mortalidad en la infancia", y la meta 5 "reducir en dos terceras partes, entre 1990 y 2015, la tasa de mortalidad de los niños menores de cinco años". Dentro de esta última se encuentra el indicador de Mortalidad Infantil medido por la tasa de muertes de niños menores de un año por cada mil nacidos vivos, en el año. El Objetivo 5 propone "mejorar la salud materna", y la meta 6 "reducir en tres cuartas partes, entre 1990 y
2015, la tasa de mortalidad derivada de la maternidad". Esta tasa o razón se refiere a las muertes debidas a causas del embarazo, parto o puerperio por cada mil nacidos vivos en el año. El denominador de nacidos vivos tiene, entonces, un carácter crucial, porque además permite calcular las coberturas de vacunación y otras tasas de morbi mortalidad.

Si bien las Encuestas han contribuido de manera notable al conocimiento de los indicadores (muchos de los cuales no se los puede conocer de otra manera), y deben ser mantenidas en el tiempo, se propone fortalecer las Estadísticas Vitales del Registro Civil, todas procesadas por el INEC, en el entendido de que es indispensable disponer de las estadísticas oficiales públicas y de los resultados de las encuestas ${ }^{2}$.

1.- REDSISMIL, Red del Sistema de Indicadores de Salud para los Objetivos del Milenio, Ecuador.

Estuardo Albán ealban@quito.inec.gov.ec César Hermida cesar.hermida@undp.org Jorge Hermida drhermida@yahoo.com Juan Vásconez jvasconez@unicef.org Mario Vergara mario.vergara@undp.org Miguel Hinojosa mahinojosa45@hotmail.com Miguel Machuca mmachuca@ecu.ops-oms.org Nelson Oviedo noviedo@cepar,org.ec

2.- Desde hace dos décadas, en el marco internacional de la "disminución del tamaño del Estado" se ha dado, en el caso del INEC y el MSP, una notable reducción de personal (desde las parroquias para el Registro Civil y las Estadísticas Vitales y del personal para procesamiento en cantones, provincias y nivel central), aparte de una disminución progresiva del valor real de los salarios. El propio Censo de 2001 tuvo dificultades de financiamiento y retraso. Esta tendencia implica el riesgo de provocar ineficiencia en las responsabilidades del Estado, deterioro de sus productos, y por lo tanto una baja calidad de la información oficial. Al mismo tiempo se ha dado una corriente que busca obtener todos los indicadores mediante encuestas apoyadas con fondos de cooperación internacional. Se trata de defender y robustecer tanto las funciones públicas como aquellas privadas, superando la tendencia a descuidar las primeras en beneficio de las segundas. Un fortalecimiento de lo público y lo privado y una fuerte interrelación de los dos parece lo más razonable. 


\section{Método:}

Se analiza la consistencia de los datos del denominador utilizando las estadísticas públicas del INEC para los nacidos vivos, así como la información del último Censo Nacional (2001). En el primer caso se suman los registros tardíos de cada año para calcular el total real, y luego se compara el número, tanto con el total de inscritos en el año, como con los datos de menores de un año del censo y sus proyecciones.

\section{Resultados:}

Los nacidos vivos inscritos a partir de 1995 constan en el Cuadro 1, con aquellos registrados en el mismo año y en los años subsiguientes. El Cuadro 2 muestra los porcentajes.

Se observa que alrededor de un $60 \%$ dnacimientos se inscriben en el mismo año, mientras un $28 \%$ lo hace en el año siguiente, y un $12 \%$ lo hace en años ulteriores $(5.5 \%$ en el segundo y el resto a partir del tercer año). Hay, por lo tanto, alrededor de un $40 \%$ de inscripción tardía en el nivel nacional.
Como el $59.63 \%$ de promedio de inscritos en el mismo año del período, es aproximado al $57.60 \%$ del promedio de las inscripciones del mismo año sobre el total de inscripciones anuales, Cuadro 3, se podría pensar que el dato de inscripciones totales puede suplir, a nivel nacional, al dato de nacidos vivos en el año ( $\mathrm{y}$ en provincias y cantones "sólo si sucediera lo mismo"), sin embargo, es menester recordar que se trata de un promedio, pues cuando se compara los números de cada año, que son los que se utilizarían, las diferencias resultan notables, Cuadro 4.

\section{Discusión:}

El denominador de "nacidos vivos" es crucial. Lamentablemente, ante la falta de ajuste y uso de las estadísticas locales, en el país se ha utilizado, a partir del Censo de 1990, un grosero estimado nacional para las necesidades locales, basado en el porcentaje de menores de un año de la población total. Así, la población nacional se redondeó a 10 millones y los menores de un año a 300 mil, dando un porcentaje de 3 \% para el estimado del denominador de nacidos Cuadro 1.

Nacidos vivos inscritos en el mismo año y en años subsiguientes Ecuador, 1995 a 2003

\begin{tabular}{|l|l|l|l|l|l|l|l|l|l|}
\hline $\begin{array}{l}\text { Naci- } \\
\text { dos } \\
\text { en: }\end{array}$ & $\begin{array}{l}\text { Inscri- } \\
\text { tos en } \\
1995\end{array}$ & 1996 & 1997 & 1998 & 1999 & 2000 & 2001 & 2002 & 2003 \\
\hline 1995 & 181268 & 90072 & 13004 & 10798 & 8351 & 5605 & 3023 & & \\
\hline 1996 & & 182242 & 88336 & 26142 & 12561 & 7265 & 4746 & 2773 & \\
\hline 1997 & & & 169869 & 101889 & 20883 & 9765 & 5474 & 4506 & 3408 \\
\hline 1998 & & & & 199079 & 76876 & 13740 & 6583 & 4467 & 4775 \\
\hline 1999 & & & & & 218108 & 87179 & 14317 & 7780 & 7774 \\
\hline $\mathbf{2 0 0 0}$ & & & & & & 202257 & 93892 & 16865 & 12426 \\
\hline $\mathbf{2 0 0 1}$ & & & & & & & 192786 & 85384 & 23243 \\
\hline $\mathbf{2 0 0 2}$ & & & & & & & & 183792 & 91508 \\
\hline $\mathbf{2 0 0 3}$ & & & & & & & & & 178549 \\
\hline
\end{tabular}

Fuente: INEC, Estadísticas Vitales (Cuadro 1: Nacidos vivos inscritos por años).

Elaboración: UNFPA Ecuador, 2004. 
Cuadro 2.

Inscripción de nacidos vivos en el mismo año y en años subsiguientes Porcentajes. Ecuador, 1995 a 2000

\begin{tabular}{|l|l|l|l|l|l|l|l|l|}
\hline Año: & $\begin{array}{l}\text { Inscri- } \\
\text { tos, } \\
\text { mismo } \\
\text { año }\end{array}$ & $\begin{array}{l}\text { Inscri- } \\
\text { tos, } \\
\text { año } \\
\text { siguiente }\end{array}$ & $\begin{array}{l}\text { Ins. 2 } \\
\text { años + } \\
\text { tarde }\end{array}$ & $\begin{array}{l}\text { Ins. 3 } \\
\text { años + } \\
\text { tarde }\end{array}$ & $\begin{array}{l}\text { Ins. 4 } \\
\text { años + } \\
\text { tarde }\end{array}$ & $\begin{array}{l}\text { Ins. 5 } \\
\text { años + } \\
\text { tarde }\end{array}$ & $\begin{array}{l}\text { Ins. 6 } \\
\text { años + } \\
\text { tarde }\end{array}$ & $\begin{array}{l}\text { Total } \\
\text { n a c i d o s } \\
\text { en el año }\end{array}$ \\
\hline 1995 & $58.07 \%$ & $28.85 \%$ & $4.16 \%$ & $3.45 \%$ & $2.67 \%$ & $1.79 \%$ & $0.96 \%$ & 312121 \\
\hline 1996 & $56.23 \%$ & $27.25 \%$ & $8.06 \%$ & $3.87 \%$ & $2.24 \%$ & $1.46 \%$ & $0.85 \%$ & 324065 \\
\hline 1997 & $53.87 \%$ & $32.31 \%$ & $6.62 \%$ & $3.09 \%$ & $1.73 \%$ & $1.42 \%$ & $0.91 \%$ & 315284 \\
\hline 1998 & $64.59 \%$ & $24.94 \%$ & $4.45 \%$ & $2.13 \%$ & $1.44 \%$ & $1.50 \%$ & $0.91 \%$ & 308206 \\
\hline 1999 & $64.18 \%$ & $25.65 \%$ & $4.21 \%$ & $2.28 \%$ & $1.46 \%$ & $1.34 \%$ & $0.84 \%$ & 339786 \\
\hline 2000 & $60.85 \%$ & $28.25 \%$ & $5.07 \%$ & $2.16 \%$ & $1.41 \%$ & $1.38 \%$ & $0.85 \%$ & 332359 \\
\hline Media & $59.63 \%$ & $27.87 \%$ & $5.42 \%$ & $2.83 \%$ & $1.82 \%$ & $1.48 \%$ & $0.88 \%$ & 323287 \\
\hline
\end{tabular}

Fuente: INEC, Estadísticas Vitales (Cuadro 1: Nacidos vivos inscritos por años).

Elaboración: UNFPA Ecuador, 2004.

NOTA: Estimados para 6 años del 98,5 y 6 del 99 y 4,5 y 6 del 2000 , calculando el promedio de los últimos ños.

Se puede concluir que un $60 \%$ se inscribe el mismo año, $28 \%$ al año siguiente y 12 en los años ulteriores

vivos. Dicha estimación se ha utilizado en los servicios locales del Ministerio de Salud por más de una década. Estimar el denominador con el número de menores de un año tiene la ventaja de obtener fácilmente el dato a partir de las proyecciones de la población, pero tiene dos graves inconvenientes: primero que el estimado no corresponde al registro de nacimientos, $y$ segundo que las proyecciones sólo se ajustan cada diez años con los censos. Por ejemplo a partir de 1990 la proyección anual de menores de un año mostraba un paulatino crecimiento, pero como se hallaba sobre estimado, al llegar a 294645 como dato censal en el 2001, se inicia, en la proyección, su declinación. Para dicho año, teniendo en cuenta la población censal total de 12479924 , el porcentaje de menores de un año corresponde al $2.36 \%$, mientras en el 2004 (con 289789 menores de un año y una población de 13026891 , ambos datos proyectados) el porcentaje es de $2.22 \%$.

El Cuadro 5 muestra que la población registrada en el Censo del 2001 como menor de un año constituye sólo el $73.37 \%$ de aquella calculada con los registros tardíos para el mismo año, y es el $80.50 \%$ de aquella estimada con las proyecciones censales. La proyección censal es incluso sólo el $91.13 \%$ de aquella calculada con los registros tardíos, pero este dato resulta clave, pues con el $8.87 \%$ de diferencia se puede estimar el número de nacidos vivos. Efectivamente, el Cuadro 6 muestra la proyección de la población de menores de un año, y la estimación con el cálculo de registros tardíos, señalando para el año 2004, por ejemplo, que el número de nacidos vivos es 315493 (es decir sumando 25704 más nacidos, $8.87 \%$ de incremento, al total de 289789 de la proyección).

\section{Conclusiones:}

Se debe calcular el registro tardío tanto para el nivel nacional como para el provincial, cantonal y parroquial, con estos ajustes es posible estimar el número de nacidos vivos para los años subsiguientes, a partir de las proyecciones censales. 
Es recomendable efectuar un ejercicio similar al propuesto para los niveles provinciales y cantonales, a fin de obtener indicadores más consistentes. En el nivel local, es decir en las ción del registro civil, para utilizarla, ajustarla y mejorarla, a fin de que la consistencia se fortalezca desde la base.

\section{Cuadro 3.}

Total de inscripciones en el año y número de nacidos en el mismos año. Porcentajes. Ecuador, 1996 a 2003

\begin{tabular}{|l|c|c|c|}
\hline Años & Inscripciones totales & Inscripciones en el mismo año & Porcentaje \\
\hline $\mathbf{1 9 9 6}$ & 302217 & 182246 & $60.30 \%$ \\
\hline $\mathbf{1 9 9 7}$ & 288803 & 169869 & $58.81 \%$ \\
\hline $\mathbf{1 9 9 8}$ & 364684 & 199079 & $54.58 \%$ \\
\hline $\mathbf{1 9 9 9}$ & 368659 & 218108 & $59.16 \%$ \\
\hline $\mathbf{2 0 0 0}$ & 345715 & 202257 & $58.50 \%$ \\
\hline $\mathbf{2 0 0 1}$ & 332776 & 192786 & $57.93 \%$ \\
\hline $\mathbf{2 0 0 2}$ & 314577 & 183792 & $58.42 \%$ \\
\hline $\mathbf{2 0 0 3}$ & 332870 & 178549 & $53.63 \%$ \\
\hline Total & 2650301 & 1526686 & $57.60 \%$ Media \\
\hline
\end{tabular}

Fuente: INEC, Estadísticas Vitales (Cuadro 1: Nacidos vivos inscritos por años).

Elaboración: UNFPA Ecuador, 2004.

parroquias, es conveniente conocer la informa-

\section{Cuadro 4}

Número total de nacidos, con inscripción tardía, e inscripciones totales en el año. Ecuador, 1996 a 2003

\begin{tabular}{|l|c|c|}
\hline Afio: & Total nacidos en el ano* & Inscripciones totales \\
\hline 1995 & 312121 & 302217 \\
\hline 1996 & 324065 & 288803 \\
\hline 1997 & 315284 & 364684 \\
\hline 1998 & 308206 & 368659 \\
\hline 1999 & 339786 & 345715 \\
\hline 2000 & 332359 & 332776 \\
\hline 2001 & 323303 & 314577 \\
\hline 2002 & $257308^{* *}$ & 332870 \\
\hline 2003 & $249968^{* *}$ & \\
\hline
\end{tabular}

* Con inscripción tardía

** Estimado agregado el $40 \%$ a los nacidos e inscritos en el mismo año. 


\section{Cuadro 5.}

Nacidos vivos totales comparados con los menores de un año del Censo del 2001 y de la Proyección Censal

Ecuador, 2001.

\begin{tabular}{|l|l|l|}
\hline $\begin{array}{l}\text { Total de Nacidos Vivos, } \\
\text { estimados con el registro } \\
\text { tardío, 2001 }\end{array}$ & 323303 & $100 \%$ \\
\hline $\begin{array}{l}\text { Estimación de la } \\
\text { población de menores de } \\
\text { un año para 2001, en base } \\
\text { y a partir del Censo } \\
\text { del 2001 }\end{array}$ & 294645 & $\begin{array}{l}\text { 91.13\% del total } \\
\text { de nacidos vivos } \\
\text { (incluyendo registro } \\
\text { tardío). }\end{array}$ \\
\hline $\begin{array}{l}\text { VI Censo de Población, 2001. } \\
\text { Población de menores } \\
\text { de un año, Total }\end{array}$ & 237209 & $\begin{array}{l}80.50 \% \text { de la estimación } \\
\text { de la población a partir } \\
\text { del censo. } \\
73.37 \% \text { del total de } \\
\end{array}$
\end{tabular}

Fuente: INEC, Estadísticas Vitales (Cuadro 1: Nacidos vivos inscritos por años).

INEC, Estimaciones y Proyecciones de Población 1950 - 2025, Serie OI No. 205, Octubre 2003.

INEC, VI Censo de Población 2001. Población de Menores de 1 año.

Elaboracion: UNFPA Ecuador, 2004.

\section{Cuadro 6}

Proyección de población menor de un año y estimación de nacidos vivos. Ecuador 2004 a 2010

\begin{tabular}{|l|l|l|}
\hline Año & Proyección Censal (1) & Estimación (2) \\
\hline 2004 & 289789 & 315493 \\
\hline 2005 & 288485 & 314073 \\
\hline 2006 & 287679 & 313196 \\
\hline 2007 & 287082 & 312546 \\
\hline 2008 & 286616 & 312038 \\
\hline 2009 & 286204 & 311590 \\
\hline 2010 & 285786 & 311135 \\
\hline
\end{tabular}

Fuentes: (1) INEC Estimaciones y proyecciones de Población, 2003.

(20 Estimaciones con $8.87 \%$ de incremento en base al cálculo de registros tardíos, UNFPA, 2004.

No es recomendable tomar el número de menores de un año y su proyección, peor el porcentaje de éstos de la población total, para reemplazarlo por el dato o estimación de naci- dos vivos. Tampoco es recomendable utilizar el número total de inscritos en el año como alternativa del número de nacidos vivos en el año. 